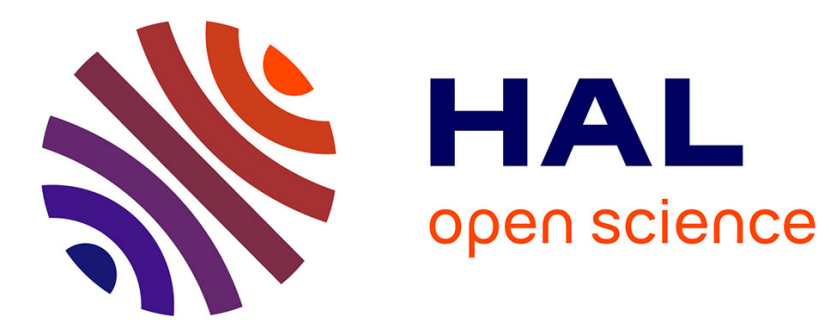

\title{
Turnpike features in optimal selection of species represented by quota models
}

Walid Djema, Laëtitia Giraldi, Sofya Maslovskaya, Olivier Bernard

\section{To cite this version:}

Walid Djema, Laëtitia Giraldi, Sofya Maslovskaya, Olivier Bernard. Turnpike features in optimal selection of species represented by quota models. Automatica, In press, 10.1016/j.automatica.2021.109804 . hal-03217983v2

\section{HAL Id: hal-03217983 \\ https://hal.science/hal-03217983v2}

Submitted on 8 Jun 2021

HAL is a multi-disciplinary open access archive for the deposit and dissemination of scientific research documents, whether they are published or not. The documents may come from teaching and research institutions in France or abroad, or from public or private research centers.
L'archive ouverte pluridisciplinaire HAL, est destinée au dépôt et à la diffusion de documents scientifiques de niveau recherche, publiés ou non, émanant des établissements d'enseignement et de recherche français ou étrangers, des laboratoires publics ou privés. 


\title{
Turnpike features in optimal selection of species represented by quota models ${ }^{\star}$
}

\author{
W. Djema ${ }^{\text {a }} \quad$ L. Giraldi $^{b} \quad$ S. Maslovskaya ${ }^{a} \quad$ O. Bernard ${ }^{\text {a,c }}$ \\ ${ }^{a}$ Université Côte d'Azur, Inria BIOCORE Project-team, France. \\ ${ }^{\mathrm{b}}$ Université Côte d'Azur (UCA), Inria Sophia Antipolis, CALISTO project-team, France. \\ ${ }^{\mathrm{c}}$ Laboratoire d'Océanographie de Villefranche (LOV), Université de Sorbonne, CNRS, Villefranche-sur-Mer, France. \\ walid.djema@inria.fr laetitia.giraldi@inria.fr sofya.maslovskaya@inria.fr olivier.bernard@inria.fr
}

\begin{abstract}
The paper focuses on a generic optimal control problem (OCP) deriving from the competition between two microbial populations in continuous cultures. The competition for nutrients is reduced to a two-dimensional dynamical nonlinear-system that can be derived from classical quota models. We investigate an OCP that achieves species separation over a fixed time-window, suitable for a large class of empirical growth functions commonly used in quota models. Using Pontryagin's Maximum Principle (PMP), the optimal control strategy steering the model trajectories is fully characterized. Then, we provide sufficient conditions for the existence of a turnpike property associated with the optimal control and state-trajectories, as well as their respective costate trajectories. Indeed, we prove that for a sufficiently large time, the optimal strategy achieving strain separation remains most of the time exponentially close to an optimal steady-state defined from an associated simpler static-OCP. This turnpike feature is based on the hyperbolicity of the linearized Hamiltonian-system around the solution of the static-OCP. The obtained theoretical results are then illustrated on microalgae, described by the Droop model in dimension 5. The optimal strategy is numerically computed in Bocop (open source toolbox for optimal control) with direct optimization methods.
\end{abstract}

Key words: Optimal control, turnpike properties, nonlinear systems, photobioreactor, strains selection, microalgae.

\section{Introduction}

Natural selection drives the fate of the species that are permanently competing for limited survival sources. The individuals with a lower fitness in local conditions will eventually disappear from the ecosystem due to the competition for survival. Combined with mutations that may appear naturally over time, the Darwinian adaptation characterizing living organisms ensures that individuals which are better tailored to their local environment are continuously emerging and then progressively dominating their surroundings. In some instances, these evolution phenomena may be guided and redirected towards different fates. It is for instance possible to modify the outcome of species competition, leading to the emergence of enhanced organisms, by controlling and modulating the natural selection pressure (Swenson (2000)). Such a selection process was then tamed for generating naturally more efficient microogranisms (Almeida et al. (2004)). Within this scope, some microalgae strains

\footnotetext{
^ Corresponding author: Walid Djema.
}

have been recently improved by setting-up a Darwinian selection pressure within artificial cultures such as photobioreactors (see, e.g., Gachelin et al. (2020); Huertas et al. (2010); Bonnefond et al. (2017)).

Microalgae attract considerable attention since they have a great potential to produce less impacting biofuels (Chisti (2007); Wijffels \& Barbosa (2010)). In addition to reducing atmospheric $\mathrm{CO}_{2}$, they are also considered as promising elemental bricks for green chemistry and profitable new protein sources. Moreover, they are even used to improve wastewater treatment (see also, Walsh et al. (2016); Odjadjare et al. (2017)). However, despite their large interest, microalgae are still in their infancy and hitherto mainly grown for cosmetic or pharmaceutical industries. Their exploitation on a larger scale, particularly for biofuel production, is conditioned by the overcoming of various technical challenges, including the necessity of enhancing the strain productivity since mainly wild species are currently cultivated. This explains the worldwide race during the last decade for 
selecting more suitable microalgae strains (see, e.g., Aravantinou et al. (2013); Rodolfi et al. (2009); Yoo et al. (2010)).

In this paper, the objective is to develop an effective control strategy ensuring that the most suitable strains will dominate the continuous artificial culture in finite time. For instance, in biofuel production, desirable microalgae strains can be those which grow faster, with higher lipid content, and which are also well adapted to local environment and seasons.

We recall that when $n$ distinct strains coexist in a chemostat with limited substrate, a well-known principle -the competitive exclusion principle CEP (Smith \& Waltman (1995))- predicts that the species requiring the smallest amount of nutrients to grow at a rate equal to the dilution rate wins the competition, while the other species will be asymptotically washed out. However, depending on the culture conditions, the winner of the competition is not in general necessarily the one with the highest lipid content or unrivalled productivity. Therefore, our goal is to determine an optimal control strategy so that the species of interest is the one that dominates in finite time. To achieve our objectives, we use an approach that is based on optimal control theory (Pontryagin's principle, Pontryagin et al. (1964)) and turnpike features (see, e.g., Trélat \& Zuazua (2015)). There are other works for optimal selection of microorganisms in minimal-time (Bayen \& Mairet (2014, 2017)), based on the simpler Monod-type dynamics describing the growth of bacteria. The microalgae behavior is however more complex since the growth of these microorganisms does not depend on the substrate concentration in the photobioreactor (like in the Monod model), but rather on the quantity of nutrients stored inside the cells (Bernard et al. (2015); Bernard (2011)). The Droop model (Droop (1968)) is widely used to represent algal growth, since it takes into account the additional dynamics governing the internal cell-quota storage (Smith \& Waltman (1995); Droop (1983)). Here we use a general form of quota models, extending the classical Droop's formulation to include different forms of growth rate. In particular, we suppose in this work that an ad'hoc controller is able to track the desired nutrient concentration in the medium. As a consequence, the classical 5 dimensional quota model (studied for instance in Djema et al. (2020a,b)) reduces to a system of dimension 2 . In addition, this work generalizes the approach in (Djema et al. $(2019,2020 \mathrm{~b})$ ), not only because it considers a generic class of systems relevant for microalgae (using different growth rates), but also because there are no assumption on the initial conditions, which are often assumed to be already confined to an attractive positively invariant set (Bayen \& Mairet (2014); Djema et al. (2019, 2020b)). The main novelty of our work is to prove the existence of a turnpike property characterizing the optimal solutions. The turnpike has been recently revisited in the optimal control literature and it is attracting more and more attention (see, e.g., Trélat \& Zhang (2018); Porretta \& Zuazua (2013); Zaslavski (2015); Trélat \& Zhang (2018); Lohéac et al. (2018); Gugat \& Hante (2019)). The turnpike property states that the optimal solutions consist of three pieces: two transient short-time arcs at the boundaries of the time interval, and a long-time behavior remaining exponentially close to a static solution (Trélat \& Zuazua (2015)). From a practical standpoint, turnpike features are also advantageous for deriving sub-optimal controls that are easy to implement on real industrial systems.

The paper is organized as follows. Sect. 2 is devoted to the statement of the general optimal control problem (OCP), formulated for a generic two-dimensional dynamical system, which includes competition between algal species. In Sect. 3 we state the main theorem dealing with the general OCP. Its proof is provided in Sect. 4, which relies on Pontryagin's maximum principle (PMP, Pontryagin et al. (1964)) and also on the existence of a turnpike property along the optimal solutions. In Sect. 5 , we focus on an application involving a variable yield model that includes the classical Droop's model. Thus, based on the results in Sect. 3-4, we solve the problem of microalgae selection under several growth-rates. Finally, in Sect. 5.5, the optimal control is determined in different cases and the turnpike feature is illustrated using a direct optimization method implemented in Bocop (optimal control toolbox, Bonnans et al. (2017)).

\section{Optimal control problem (OCP)}

We consider a generic nonlinear two-dimensional dynamical system, suitable for competition between two populations in which the dynamics are coupled through the feeding control-flow. Typically, the competition between two species (e.g., phytoplankton, bacteria, yeasts,...) for a limiting substrate $(w)$, whose concentration is controlled in a continuous bioreactor, can be represented as follows:

$$
\left\{\begin{array}{l}
\dot{y}_{1}=-f_{1}\left(y_{1}\right) y_{1}+g_{1}(w), \\
\dot{y}_{2}=-f_{2}\left(y_{2}\right) y_{2}+g_{2}(w),
\end{array}\right.
$$

where the $f_{i}(\cdot)$ are regular nonnegative real-valued and increasing functions representing the growth rate of species $i$ with respect to the intracellular limiting nutrient $y_{i}$. The functions $g_{i}(\cdot)$ are also regular nonnegative real-valued and increasing standing for the flux of limiting nutrient into the cell. System (1) typically appears as a subsystem of quota models, where $y_{i}$ is the internal quota of the $i$-th species, i.e. the intracellular amount of the element for which the involved species are competing. The Droop's model is extensively studied later in Sect. 5 as the most famous example of this class of systems. However, the model (1) can also be found in more complicated metabolic dynamical systems (Wiechert (2002)) where the intracellular element $y_{i}$ is diluted by growth. The resulting system (1) is positive, i.e., for positive initial conditions the trajectories remain positive. 
In general, the dynamics of the system (1) is at higher dimension ( 5 in the case of Droop's model), but here we assume that the limiting substrate has been controlled by an ad'hoc controller tracking the concentration $w(t)$. Indeed, the way this subsystem can be extracted from the full model is developed in Sect. 5 in the typical case of Droop's model describing microalgae. Without loss of generality, we consider throughout this paper that species 1 is the one of interest and must become predominant in the population in finite time. Our objective is to investigate the control strategies for this competition between two initially co-existing populations by controlling the nonnegative and bounded input $w$ over a fixed time-interval $\left[0, T_{f}\right]$.

\subsection{Statement of the general OCP}

The set of admissible controls associated with system (1) is defined by $w \in \mathcal{W}$, s.t., $\mathcal{W}$ is a subset of $\mathcal{L}_{\text {loc }}^{\infty}\left(\mathbb{R}^{+}\right)$, the space of locally integrable functions on every compact set on $\mathbb{R}^{+}$. More precisely, we set,

$w \in \mathcal{W}=\left\{w:\left[0, T_{f}\right] \rightarrow\left[0, w_{m}\right] \mid w(\cdot) \in \mathcal{L}_{\text {loc }}^{\infty}\left(\mathbb{R}^{+}\right)\right\}$,

where $w_{m}$ is a sufficiently large positive constant (the maximum influent substrate concentration). Then, we define now the main OCP that reads,

$\left\{\begin{array}{c}\underset{w \in \mathcal{W}}{\operatorname{Maximize}} \mathcal{C}_{\left[0, T_{f}\right]}=\int_{0}^{T_{f}} f_{1}\left(y_{1}(\ell)\right)-f_{2}\left(y_{2}(\ell)\right) d \ell \\ \text { where, } y=\left(y_{1}, y_{2}\right), y(\cdot) \text { is the solution of }(1) \\ \text { corresponding to the control } w(\cdot) .\end{array}\right.$

In what follows, the terminal conditions of the states $y_{i}, i=1,2$, are assumed to be free and their initial conditions are positive. Besides, let us define $\left(y_{i}^{*}, w^{*}\right)$, $i=1,2$, the steady state of (1), where, $y_{i}^{*}>0$ and $w^{*} \in\left[0, w_{m}\right]$. It follows that, $f_{i}\left(y_{i}^{*}\right) y_{i}^{*}=g_{i}\left(w^{*}\right)$, for $i=1,2$, and since the map, $y_{i} \rightarrow f_{i}\left(y_{i}\right) y_{i}$, is increasing, we get, $w^{*}=\delta_{i}\left(y^{*}\right)$, where, $\delta_{i}(y)=g_{i}^{-1}\left(f_{i}\left(y_{i}\right) y_{i}\right)$. Thus, $y_{i}^{*}\left(w^{*}\right)=\delta_{i}^{-1}\left(w^{*}\right), i=1,2$, for all fixed $w^{*} \in\left[0, w_{m}\right]$. Then now, we define for all admissible $y_{i}^{*}>0$ and $w^{*} \in$ $\left[0, w_{m}\right]$ the function,

$$
\Delta\left(w^{*}\right)=f_{1}\left(\delta_{1}^{-1}\left(w^{*}\right)\right)-f_{2}\left(\delta_{2}^{-1}\left(w^{*}\right)\right)
$$

Throughout this work, we assume that the maximizer of $\Delta$ is the unique point $\bar{w} \in\left(0, w_{m}\right)$, as illustrated in Fig. 1 . The cases where $\bar{w}$ is zero or $w_{m}$ are pointless and can be trivially addressed outside this framework. Let us remark that under these considered assumptions, OCP (2) has a maximizer using Filippov Theorem (see, e.g., Cesari (1983)). In fact, the generic form of the functions $f_{i}\left(\delta_{i}^{-1}(\cdot)\right)$, involving one intersection point over $\left(0, w_{m}\right)$ as in Fig. 1, ensures the existence of control functions maximizing and minimizing the criterion $\mathcal{C}_{\left[0, T_{f}\right]}$, as a direct consequence of the competitive exclusion principle (CEP), (see, Smith \& Waltman (1995)).
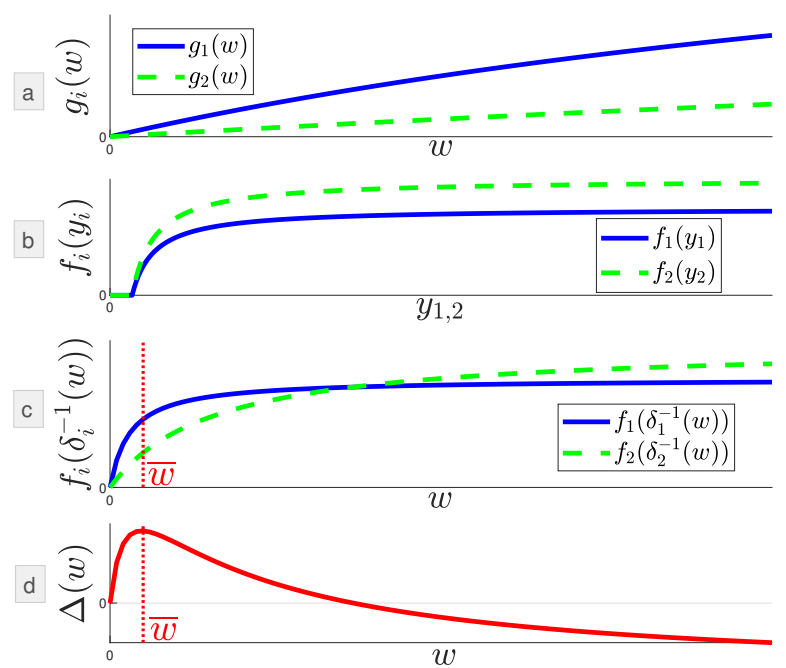

Fig. 1. (a-b) provide the generic form of the functions $f_{i}$ and $g_{i}$ for $i=1,2$ (with arbitrary units), studied in this paper. (c) illustrates the resulting $f_{i}\left(\delta_{i}^{-1}(w)\right)$, in their typical general form studied throughout this work, where both situations $f_{1}\left(\delta_{1}^{-1}(w)\right)>f_{2}\left(\delta_{2}^{-1}(w)\right)$ and $f_{1}\left(\delta_{1}^{-1}(w)\right)<f_{2}\left(\delta_{2}^{-1}(w)\right)$ hold over $\left[0, w_{m}\right]$. The resulting function $\Delta$ in $(\mathbf{d})$ satisfies the hypothesis $\bar{w} \in\left(0, w_{m}\right)$.

\subsection{Pontryagin's maximum principle (PMP)}

The Hamiltonian associated with OCP (2) is defined by,

$$
\begin{aligned}
H= & \Phi\left(\lambda_{y_{1}}, \lambda_{y_{2}}, w\right)-\lambda_{y_{1}} f_{1}\left(y_{1}\right) y_{1}-\lambda_{y_{2}} f_{2}\left(y_{2}\right) y_{2} \\
& +\lambda_{0}\left[f_{1}\left(y_{1}\right)-f_{2}\left(y_{2}\right)\right]
\end{aligned}
$$

where,

$$
\Phi\left(\lambda_{y_{1}}, \lambda_{y_{2}}, w\right)=\lambda_{y_{1}} g_{1}(w)+\lambda_{y_{2}} g_{2}(w) .
$$

It is classical to consider that $\lambda_{0}=1$ since OCP (2) is stated as a maximization problem. The PMP implies the existence of absolutely-continuous $\lambda_{y_{i}}$, which are the costate of $y_{i}$, satisfying, $\dot{\lambda}_{y_{i}}=-\partial H / \partial y_{i}$, for $i=1$, 2 . In addition, since the final states $y_{i}\left(T_{f}\right)$ are free, it follows that the transversality conditions are given by,

$$
\lambda_{y_{i}}\left(T_{f}\right)=0, \quad \text { for } \quad i=1,2 .
$$

Finally, the PMP implies that the control function $w \in$ $\mathcal{W}$ satisfies, for almost all $t \in\left[0, T_{f}\right]$,

$$
\max _{w(t) \in\left[0, w_{m}\right]} H\left(y_{i}(t), \lambda_{y_{i}}(t), w(t)\right) .
$$

\subsection{Introducing the static-OCP}

The static-OCP (see, e.g., Trélat \& Zuazua (2015)) associated with the OCP (2), for the system at steady state is:

$\left\{\begin{array}{l}\underset{w \in\left[0, w_{m}\right]}{\operatorname{Maximize}} \Delta(w)=f_{1}\left(y_{1}(w)\right)-f_{2}\left(y_{2}(w)\right), \\ \text { under the constraints } f_{i}\left(y_{i}\right) y_{i}=g_{i}(w), i=1,2\end{array}\right.$ 
In other words, the static-OCP seeks to maximize the function $\Delta$ defined in (3) when system (1) is at steady state. Under the assumption that $\bar{w} \in\left(0, w_{m}\right)$ is the unique point maximizing $\Delta$, as in Fig. 1, the definition of the static-OCP is extended to include the co-state variables using the Hamiltonian equation. Indeed, according to the Lagrange multipliers rule, there exists, $\left(\bar{\lambda}_{y_{1}}, \bar{\lambda}_{y_{2}}\right) \in \mathbb{R}^{2}$, s.t., the solution $\left(\bar{y}_{1}, \bar{y}_{2}, \bar{w}\right)$, implies that $\left(\bar{y}_{1}, \bar{y}_{2}, \bar{\lambda}_{y_{1}}, \bar{\lambda}_{y_{2}}, \bar{w}\right)$ also satisfies:

$$
\left\{\begin{array}{l}
\frac{\partial H}{\partial \lambda_{y_{i}}}\left(\bar{y}_{1}, \bar{y}_{2}, \bar{\lambda}_{y_{1}}, \bar{\lambda}_{y_{2}},-1, \bar{w}\right)=0 \\
-\frac{\partial H}{\partial y_{i}}\left(\bar{y}_{1}, \bar{y}_{2}, \bar{\lambda}_{y_{1}}, \bar{\lambda}_{y_{2}},-1, \bar{w}\right)=0 \\
\frac{\partial H}{\partial w}\left(\bar{y}_{1}, \bar{y}_{2}, \bar{\lambda}_{y_{1}}, \bar{\lambda}_{y_{2}},-1, \bar{w}\right)=0
\end{array}\right.
$$

More precisely, using $H$ given in (4) we deduce that the steady-state satisfying the static-OCP (8) is solution of,

$$
\left\{\begin{array}{l}
-f_{i}\left(\bar{y}_{i}\right) \bar{y}_{i}+g_{i}(\bar{w})=0, \quad i=1,2 \\
\bar{\lambda}_{y_{1}}\left(f_{1}\left(\bar{y}_{1}\right)+f_{1}^{\prime}\left(\bar{y}_{1}\right) \bar{y}_{1}\right)-f_{1}^{\prime}\left(\bar{y}_{1}\right)=0 \\
\bar{\lambda}_{y_{2}}\left(f_{2}\left(\bar{y}_{2}\right)+f_{2}^{\prime}\left(\bar{y}_{2}\right) \bar{y}_{2}\right)+f_{2}^{\prime}\left(y_{2}\right)=0 \\
g_{1}^{\prime}(\bar{w}) \bar{\lambda}_{y_{1}}+g_{2}^{\prime}(\bar{w}) \bar{\lambda}_{y_{2}}=0
\end{array}\right.
$$

In the general case illustrated in Fig. 1, we define the unique static optimal steady-state that satisfies (10), denoted by,

$$
\mathcal{E}^{\text {static }}=\left(\bar{w}, \bar{y}_{1}, \bar{y}_{2}, \bar{\lambda}_{y_{1}}, \bar{\lambda}_{y_{2}}\right) .
$$

In Sect. 5, an explicit determination of $\mathcal{E}^{\text {static }}$ is given for typical $f_{i}$ and $g_{i}$ functions in quota models.

\section{$3 \quad$ Turnpike main result : Theorem 1}

Let us consider the perturbed trajectories around the static optimal steady-state $\mathcal{E}^{\text {static }}$ defined in (11). More precisely, we perform the classical change of coordinates, $\delta y_{i}(t)=y_{i}(t)-\bar{y}_{i}, \delta \lambda_{y_{i}}(t)=\lambda_{y_{i}}(t)-\bar{\lambda}_{y_{i}}$, for $i=1,2$, and we consider the shifted control, $\delta w(t)=w(t)-\bar{w}$. Thus, as in (Trélat \& Zuazua (2015)), we can check that, $\delta w(t)=-H_{w w}^{-1}\left(H_{w y} \delta y(t)+H_{w \lambda} \delta \lambda_{y}(t)\right)$, where, $\delta y=\left(\delta y_{1}, \delta y_{2}\right)^{\mathrm{T}}$ and $\delta \lambda_{y}=\left(\delta \lambda_{y_{1}}, \delta \lambda_{y_{2}}\right)^{\mathrm{T}}$.

In what follows, we set $z=\left(\delta y, \delta \lambda_{y}\right)^{\mathrm{T}}$.

Using the Hamiltonian (4), we notice that $H_{y w}=$ $\left.\frac{\partial^{2} H}{\partial y \partial w}\right|_{\mathcal{E}^{\text {static }}}=(0,0)$, and thus around the unique point $\mathcal{E}^{\text {static }}$ we end up with,

$$
\left(\begin{array}{c}
\delta \dot{y}(t) \\
\delta \dot{\lambda}_{y}(t)
\end{array}\right)=\Psi\left(\begin{array}{c}
\delta y(t) \\
\delta \lambda_{y}(t)
\end{array}\right)+\left(\begin{array}{c}
\Lambda_{1}\left(\delta y(t), \delta \lambda_{y}(t)\right) \\
\Lambda_{2}\left(\delta y(t), \delta \lambda_{y}(t)\right)
\end{array}\right)
$$

for all $t \in\left[0, T_{f}\right]$, where $\Lambda_{i}$ satisfies,

$$
\frac{\left\|\Lambda_{i}\left(\delta y, \delta \lambda_{y}\right)\right\|}{\left\|\left(\delta y, \delta \lambda_{y}\right)\right\|} \underset{\left(\delta y, \delta \lambda_{y}\right) \rightarrow 0}{\longrightarrow} 0 .
$$

and,

$$
\Psi=\left(\begin{array}{cc}
H_{\lambda y} & -H_{\lambda w} H_{w w}^{-1} H_{w \lambda} \\
-H_{y y} & -H_{y \lambda}
\end{array}\right)
$$

where,

$$
\begin{gathered}
H_{w w}=\left.\frac{\partial^{2} H}{\partial w^{2}}\right|_{\mathcal{E}^{\text {static }}}=\bar{\lambda}_{y_{1}} g_{1}^{\prime \prime}(\bar{w})+\bar{\lambda}_{y_{2}} g_{2}^{\prime \prime}(\bar{w}), \\
H_{y y}=\operatorname{diag}\left\{-\bar{\lambda}_{y_{1}}\left(2 f_{1}^{\prime}\left(\bar{y}_{1}\right)+\bar{y}_{1} f_{1}^{\prime \prime}\left(\bar{y}_{1}\right)\right)+f_{1}^{\prime \prime}\left(\bar{y}_{1}\right),\right. \\
\left.-\bar{\lambda}_{y_{2}}\left(2 f_{2}^{\prime}\left(\bar{y}_{2}\right)+\bar{y}_{2} f_{2}^{\prime \prime}\left(\bar{y}_{2}\right)\right)-f_{2}^{\prime \prime}\left(\bar{y}_{2}\right)\right\}, \\
H_{\lambda y}=\operatorname{diag}\left\{-f_{1}\left(\bar{y}_{1}\right)-\bar{y}_{1} f_{1}^{\prime}\left(\bar{y}_{1}\right),-f_{2}\left(\bar{y}_{2}\right)-\bar{y}_{2} f_{2}^{\prime}\left(\bar{y}_{2}\right)\right\} \\
H_{w \lambda}=\left(g_{1}^{\prime}(\bar{w}), g_{2}^{\prime}(\bar{w})\right), \text { and thus we end up with },
\end{gathered}
$$

$$
H_{\lambda w} H_{w w}^{-1} H_{w \lambda}=\left(\begin{array}{cc}
\frac{g_{1}^{\prime 2}(w)}{\lambda_{y_{1}} g_{1}^{\prime \prime}(w)+\lambda_{y_{2}} g_{2}^{\prime \prime}(w)} & \frac{g_{1}^{\prime}(w) g_{2}^{\prime}(w)}{\lambda_{y_{1}} g_{1}^{\prime \prime}(w)+\lambda_{y_{2}} g_{2}^{\prime \prime}(w)} \\
\frac{g_{1}^{\prime}(w) g_{2}^{\prime}(w)}{\lambda_{y_{1}} g_{1}^{\prime \prime}(w)+\lambda_{y_{2}} g_{2}^{\prime \prime}(w)} & \frac{g_{2}^{\prime 2}(w)}{\lambda_{y_{1}} g_{1}^{\prime \prime}(w)+\lambda_{y_{2}} g_{2}^{\prime \prime}(w)}
\end{array}\right) .
$$

Let us define the following matrices and constants, $-H_{\lambda w} H_{w w}^{-1} H_{w \lambda} H_{y y}=\left(\begin{array}{ll}\mathfrak{b}_{1} & \mathfrak{b}_{2} \\ \mathfrak{b}_{3} & \mathfrak{b}_{4}\end{array}\right), \mathfrak{b}_{23}=\mathfrak{b}_{2} \mathfrak{b}_{3}, \mathfrak{a}_{i}=$ $-\left.\frac{\partial\left(f_{i}\left(y_{i}\right) y_{i}\right)}{\partial y_{i}}\right|_{\mathcal{E}^{\text {static }}}, H_{\lambda y}=\operatorname{diag}\left\{\mathfrak{a}_{1}, \mathfrak{a}_{2}\right\}$. Consequently, we can show that the eigenvalues $\kappa_{j}, j=1, \ldots, 4$, of the matrix $\Psi$ defined in (14) are given by,

$$
\begin{array}{ll}
\kappa_{1}=\frac{1}{\sqrt{2}} \sqrt{\mathfrak{a}_{1}^{2}+\mathfrak{a}_{2}^{2}-\mathfrak{b}_{1}-\mathfrak{b}_{4}+\varsigma^{1 / 2}}, \quad \kappa_{2}=-\kappa_{1}, \\
\kappa_{3}=\frac{1}{\sqrt{2}} \sqrt{\mathfrak{a}_{1}^{2}+\mathfrak{a}_{2}^{2}-\mathfrak{b}_{1}-\mathfrak{b}_{4}-\varsigma^{1 / 2}}, \quad \kappa_{4}=-\kappa_{3},
\end{array}
$$

where, $\varsigma=\left[\left(\mathfrak{a}_{1}^{2}-\mathfrak{a}_{2}^{2}\right)-\left(\mathfrak{b}_{1}-\mathfrak{b}_{2}\right)\right]^{2}-4 \mathfrak{b}_{23}$.

Now, we are ready to state the main result of the paper.

Theorem 1 Let $T_{f}>0$ be a large fixed final-time and $\mathcal{E}^{\text {static }}$ be the unique global solution of the static-OCP (8), s.t. $\bar{w} \in\left(0, w_{m}\right)$ maximizes $\Delta$ in $(3)$.

(1) The solution of $O C P(2)$ with fixed initial conditions $y_{i}(0) \in \mathbb{R}_{+}^{*}, i=1,2$, correspond to the singular control function $w_{s} \in \mathcal{W}$, s.t.,

$$
w_{s}(t) \neq\left\{0, w_{m}\right\}, \text { for all } t \in\left[0, T_{f}\right]
$$

i.e., the optimal control maximizing $H$ in (4) is neither a bang 0 nor a bang $w_{m}$.

(2) Moreover, if $\mathfrak{a}_{1} \neq \mathfrak{a}_{2}, H_{w w}<0$, and,

$$
\operatorname{Re}\left(\kappa_{j}\right) \neq 0, \text { for } j=1, \ldots, 4,
$$

where $\kappa_{j}$ are the eigenvalues of the matrix $\Psi$ given in (15), then there exist positive constants $\epsilon, c_{1}, c_{2}$ and a time-instant $T^{*}>0$, s.t., for all $T_{f}>T^{*}$, if,

$$
\sum_{i=1}^{2}\left\|y_{i}(0)-\bar{y}_{i}\right\|+\left\|\bar{\lambda}_{y_{i}}\right\|<\epsilon
$$


then the optimal solutions of $O C P(2)$ satisfy $\forall t \in\left[0, T_{f}\right]$,

$$
\begin{aligned}
& \sum_{i=1}^{2}\left\|y_{i}(t)-\bar{y}_{i}\right\|+\left\|\lambda_{y_{i}}(t)-\bar{\lambda}_{y_{i}}\right\| \\
& +\left\|w_{s}(t)-\bar{w}\right\| \leq c_{1}\left(e^{-c_{2} t}+e^{-c_{2}\left(T_{f}-t\right)}\right)
\end{aligned}
$$

where $c_{1}$ does not depend on $T_{f}$ and the optimal control is the singular $w_{s}$ in (1).

Remark 1 Assuming that $T_{f}$ is sufficiently large is classical in turnpike framework (Trélat \& Zuazua (2015)). The proof of Theorem 1 provides an estimate on the lower-bound of $T_{f}$. This bound is satisfied in biological applications, e.g., metabolite production (Yegorov et al. (2019); Caillau et al. (2020)), algal growth (Djema et al. (2020b)) due to the selection time which is much larger than the time-constants characterizing the system $d y$ namics.

Remark 2 The conditions (17) ensure that the real part of the eigenvalues of the matrix $\Psi$ are non-zero (i.e. they are not on the imaginary axis), which leads to a hyperbolic behavior around the static steady-state. In practice, the conditions (17) are satisfied for a large class of systems describing microalgae growth, as illustrated in Sect. 5.5.

Remark 3 In the proof of the second part of Theorem 1, we use the approach given in Pighin \& Porretta (2016); Trélat \& Zuazua (2015). Notice that the main theorem from Trélat \& Zuazua (2015) cannot be applied to our class of optimal control problems. Firstly, the control is bounded in the considered class of optimal control problem whereas it is unbounded in Trélat \& Zuazua (2015). Secondly, the matrix $-H_{y y}+H_{y w} H_{w w}^{-1} H_{w y}$ is not positive definite in our case. For instance, choosing $f_{i}\left(y_{i}\right)=$ $\frac{a_{i} y_{i}-b_{i}}{y_{i}}$, it follows that $2 f_{1}^{\prime}\left(y_{1}\right)+y_{1} f_{1}^{\prime \prime}\left(y_{1}\right)=0$, and then we deduce that, $-H_{y y}+H_{y w} H_{w w}^{-1} H_{w y}=-H_{y y}=$ $\operatorname{diag}\left\{f_{1}^{\prime \prime}\left(\bar{y}_{1}\right),-f_{2}^{\prime \prime}\left(\bar{y}_{2}\right)\right\}$, which does not satisfy the positive definite condition in Trélat \& Zuazua (2015). This point is illustrated numerically in Sect. 5.5 using Droop's functions. Therefore, the hypotheses of the second part of Theorem 1 are slightly different from the hypotheses of the main theorem in Trélat \& Zuazua (2015).

The proof of Theorem 1 is divided in two parts. The first step concerns the statement (1) and derives from the PMP. The second step is based on the hyperbolicity of the linearized system $\dot{z}=\Psi z+\Lambda(z)$, where $z=(\delta y, \delta \lambda y)^{T}$. The arguments similar to those in Pighin \& Porretta (2016); Trélat \& Zuazua (2015) are sketched, the proof is detailed in the associated technical report: Djema et al. (2021).

\section{Proof of Theorem 1}

(1) Using (4)-(5), we note that the PMP implies that the admissible control function satisfies, for almost all $t \in\left[0, T_{f}\right]$, the maximization condition,

$$
\max _{w(t) \in\left[0, w_{m}\right]} H\left(y_{i}, \lambda_{y_{i}}, w\right)=\max _{w(t) \in\left[0, w_{m}\right]} \Phi\left(\lambda_{y_{i}}, w\right) .
$$

At first sight, we deduce from (20) that any extremal solution should be a concatenation of bang and singular arcs over $\left[0, T_{f}\right]$. Indeed, using $(5)$ we notice that in the case where $\lambda_{y_{1}}(t) \geq 0$ and $\lambda_{y_{2}}(t) \geq 0$, the control satisfying $(20)$ is the bang $w(t)=w_{m}$. Similarly, if $\lambda_{y_{1}}(t) \leq 0$ and $\lambda_{y_{2}}(t) \leq 0$, then it follows that the control satisfying $(20)$ is the bang $w(t)=0$. And finally, when $\lambda_{y_{1}}(t) \lambda_{y_{2}}(t)<0$ at any $t \in\left[0, T_{f}\right]$, we deduce from the fact that $g_{i}$ is a positive increasing function that the control satisfying (20) is the singular control denoted by $w_{s}(t)$, which maximizes $\Phi$ and which is neither 0 nor $w_{m}$. However, using the co-states dynamics given by,

$$
\left\{\begin{array}{l}
\dot{\lambda}_{y_{1}}=\lambda_{y_{1}}\left(f_{1}\left(y_{1}\right)+f_{1}^{\prime}\left(y_{1}\right) y_{1}\right)-f_{1}^{\prime}\left(y_{1}\right) \\
\dot{\lambda}_{y_{2}}=\lambda_{y_{2}}\left(f_{2}\left(y_{2}\right)+f_{2}^{\prime}\left(y_{2}\right) y_{2}\right)+f_{2}^{\prime}\left(y_{2}\right)
\end{array}\right.
$$

where $f_{i}$ are continuously increasing, we deduce that, $\bar{\lambda}_{y_{1}}=\frac{f_{1}^{\prime}\left(\bar{y}_{1}\right)}{f_{1}\left(\bar{y}_{1}\right)+f_{1}^{\prime}\left(\bar{y}_{1}\right) \bar{y}_{1}}>0$ and $\bar{\lambda}_{y_{2}}=\frac{-f_{2}^{\prime}\left(\bar{y}_{2}\right)}{f_{2}\left(\bar{y}_{2}\right)+f_{2}^{\prime}\left(\bar{y}_{2}\right) \bar{y}_{2}}<0$. Then, since $\bar{\lambda}_{y_{i}}$ is an equilibrium point of (21) and using the transversality conditions (6), we deduce that,

$$
\left\{\begin{array}{l}
0<\lambda_{y_{1}}(t)<\bar{\lambda}_{y_{1}} \\
\bar{\lambda}_{y_{2}}<\lambda_{y_{2}}(t)<0
\end{array}\right.
$$

for all $t \in\left[0, T_{f}\right)$, where the latter inequalities derive from the fact that $\partial\left(f_{i}\left(y_{i}\right) y_{i}\right) / \partial y_{i}>0$ and $f_{i}^{\prime}\left(y_{i}\right)>0$ in (21). In particular, (22) imply that for all $t \in\left[0, T_{f}\right)$,

$$
\lambda_{y_{1}}(t) \lambda_{y_{2}}(t)<0 \text {. }
$$

Consequently, we conclude from (23) that the control $w_{s}(t)$ is the one that satisfies the PMP and thus steers over the time window $\left[0, T_{f}\right]$ the trajectories from $y_{1}(0)$, $y_{2}(0)$ to $y_{1}\left(T_{f}\right), y_{2}\left(T_{f}\right)$. This concludes the proof of (1).

(2) Now, let us focus on the eigenvalues of the matrix $\Psi$ defined in (14). Firstly, let us observe that,

$$
\operatorname{det}\left(\Psi-\kappa I_{4}\right)=\operatorname{det}\left(\begin{array}{cc}
H_{\lambda y}-\kappa I_{2} & -H_{\lambda w} H_{w w}^{-1} H_{w \lambda} \\
-H_{y y} & -H_{y \lambda}-\kappa I_{2}
\end{array}\right)
$$

where $I_{\mathfrak{p}}$ is the $\mathfrak{p} \times \mathfrak{p}$ identity matrix. We notice that $H_{y y}$ and $-H_{y \lambda}-\kappa I_{2}$ are diagonal and thus they are commutative. Therefore, it follows that,

$$
\begin{aligned}
& \operatorname{det}\left(\Psi-\kappa I_{4}\right)= \\
& \quad \operatorname{det}\left(\left(H_{\lambda y}-\kappa I_{2}\right)\left(-H_{\lambda y}-\kappa I_{2}\right)-H_{\lambda w} H_{w w}^{-1} H_{w \lambda} H_{y y}\right) .
\end{aligned}
$$

Consequently, $\operatorname{det}\left(\Psi-\kappa I_{4}\right)=0$, leads to,

$$
\left(\mathfrak{a}_{1}^{2}-\kappa^{2}-\mathfrak{b}_{1}\right)\left(\mathfrak{a}_{2}^{2}-\kappa^{2}-\mathfrak{b}_{4}\right)-\mathfrak{b}_{23}=0,
$$


where the constants $\mathfrak{a}$ 's and $\mathfrak{b}$ 's are defined before Theorem 1 . From the equation (24) we readily get the eigenvalues $\kappa_{j}, j=1, \ldots, 4$, given in (15). Under the suitable assumptions expressed in (17), we ensure that the studied optimality system is hyperbolic, i.e., all eigenvalues of $\Psi$ have nonzero real parts. Next, by Theorem 6 of Molinari (1977), it follows that there exist symmetric $2 \times 2$ matrices $P_{-}, P_{+}$maximal and minimal solutions of the Riccati equation,

$$
H_{y \lambda} P+P H_{\lambda y}-H_{y y}-P H_{\lambda w} H_{w w}^{-1} H_{w \lambda} P=0 .
$$

Moreover, the corresponding matrices $\Omega_{+}=H_{\lambda y}-$ $H_{\lambda w} H_{w w}^{-1} H_{w \lambda} P_{+}$and $\Omega_{-}=H_{\lambda y}-H_{\lambda w} H_{w w}^{-1} H_{w \lambda} P_{-}$ are such that eigenvalues of $\Omega_{+}$have strictly positive real part and eigenvalues of $\Omega_{-}$have strictly negative real part, i.e., $\Omega_{+}$is unstable and $\Omega_{-}$is stable. We denote by, $V:=\left(\begin{array}{cc}I & I \\ P_{-} & P_{+}\end{array}\right)$, where $I$ is the $2 \times 2$ identity matrix. Straightforward calculations show that $\tilde{\Psi}=V^{-1} \Psi V=\operatorname{diag}\left\{\Omega_{-}, \Omega_{+}\right\}$. We denote by $\tilde{z}$ the new variable s.t. $V \tilde{z}=z$, and then it follows from (12) that,

$$
\left\{\begin{array}{l}
\dot{\tilde{z}}_{1}(t)=\Omega_{-} \tilde{z}_{1}(t)+\theta_{1}\left(\tilde{z}_{1}(t), \tilde{z}_{2}(t)\right) \\
\dot{\tilde{z}}_{2}(t)=\Omega_{+} \tilde{z}_{2}(t)+\theta_{2}\left(\tilde{z}_{1}(t), \tilde{z}_{2}(t)\right)
\end{array}\right.
$$

where the $\theta_{i}, i=1,2$, satisfy the o-little property as in (13). At this juncture, we point out that under different hypothesis, we have rewritten the optimality conditions in the form (25) similarly to Trélat \& Zuazua (2015) and Pighin \& Porretta (2016). It can be shown (see Lemmas 2-3, Djema et al. (2021)), that there exists $\mathfrak{r}>0$ s.t. for all $\left(\tilde{z}_{1}^{0}, \tilde{z}_{2}^{f}\right)$ satisfying, $\tilde{z}_{1}^{0}=\tilde{z}_{1}(0), \quad \tilde{z}_{1}^{f}=$ $\tilde{z}_{2}\left(T_{f}\right), \quad\left(\tilde{z}_{1}^{0}, \tilde{z}_{2}^{f}\right) \in B(0, \mathfrak{r})$, the corresponding solution $\left(\tilde{z}_{1}\left(t,\left(\tilde{z}_{1}^{0}, \tilde{z}_{2}^{f}\right), \tilde{z}_{2}\left(t,\left(\tilde{z}_{1}^{0}, \tilde{z}_{2}^{f}\right)\right)\right.\right.$ of system $(25)$ admits for any $t \in\left[0, T_{f}\right]$ the bound,

$$
\left\{\begin{array}{l}
\left\|\tilde{z}_{1}(t)\right\| \leq C_{\kappa}\left[\left\|\tilde{z}_{1}^{0}\right\| e^{-\kappa t}+e^{-\kappa\left(T_{f}-t\right)}\left\|\tilde{z}_{2}^{f}\right\| \Theta_{1}\left(\left\|\tilde{z}_{1}\right\|_{c^{0}}\right)\right] \\
\left\|\tilde{z}_{2}(t)\right\| \leq C_{\kappa}\left[\left\|\tilde{z}_{2}^{f}\right\| e^{-\kappa\left(T_{f}-t\right)}+e^{-\kappa t}\left\|\tilde{z}_{1}^{0}\right\| \Theta_{2}\left(\left\|\tilde{z}_{2}\right\|_{c^{0}}\right)\right]
\end{array}\right.
$$

Next step is to verify that there exist $T^{*}>0$ and $\epsilon>0$ in (18) s.t., for any $T_{f}>T^{*}$ and $y(0)$ satisfying (18), the initial and final conditions $\left(\tilde{z}_{1}^{0}, \tilde{z}_{2}^{f}\right)$ satisfy,

$$
\left\{\begin{array}{l}
\tilde{z}_{1}^{0}+\tilde{z}_{2}(0)=y_{0}-\bar{y} \\
P_{-} \tilde{z}_{1}\left(T_{f}\right)+P_{+} \tilde{z}_{2}^{f}=-\bar{\lambda}_{y}
\end{array}\right.
$$

For that, we use the fixed point argument as in Pighin $\&$ Porretta (2016), by defining the map $F: B(0, \mathfrak{r}) \rightarrow$ $\mathbb{R}^{4}$, s.t.

$$
F\left(\begin{array}{c}
\tilde{z}_{1}^{0} \\
\tilde{z}_{2}^{f}
\end{array}\right)=\left(\begin{array}{c}
y_{0}-\bar{y}-\tilde{z}_{2}(0) \\
-P_{+}^{-1}\left(P_{-} \tilde{z}_{1}\left(T_{f}\right)+\bar{\lambda}_{y}\right)
\end{array}\right)
$$

and showing that $F$ admits the unique fixed point $\left(\tilde{z}_{1}^{0}, \tilde{z}_{2}^{f}\right)$. This step is detailed in Appendix D.4 in Djema et al. (2021). It is worth mentioning that at this stage of the proof, we also get the lower-bound $T^{*}$ of $T_{f}$ and $\epsilon$ from (18). Finally, using (26) for $T_{f}>T^{*}$, we obtain for all $t \in\left[0, T_{f}\right]$,

$$
\begin{aligned}
\|\delta y(t)\|= & \left\|\tilde{z}_{1}\left(t,\left(\tilde{z}_{1}^{0}, \tilde{z}_{2}^{f}\right)\right)\right\|+\left\|\tilde{z}_{2}\left(t,\left(\tilde{z}_{1}^{0}, \tilde{z}_{2}^{f}\right)\right)\right\| \\
\leq & C_{\kappa}\left(\left\|\tilde{z}_{1}^{0}\right\| e^{-\kappa t}+e^{-\kappa\left(T_{f}-t\right)}\left\|\tilde{z}_{2}^{f}\right\| \Theta_{1}(\mathfrak{r})\right) \\
& +C_{\kappa}\left(\left\|\tilde{z}_{2}^{f}\right\| e^{-\kappa\left(T_{f}-t\right)}+e^{-\kappa t}\left\|\tilde{z}_{1}^{0}\right\| \Theta_{2}(\mathfrak{r})\right) .
\end{aligned}
$$

Up to defining a bigger constant $C_{\kappa}$, we deduce that,

$$
\|\delta y(t)\| \leq C_{\kappa}\left(e^{-\kappa t}+e^{-\kappa\left(T_{f}-t\right)}\right), \quad \forall t \in\left[0, T_{f}\right]
$$

Note that $C_{\kappa}$ is independent of $T_{f}$. Similarly, we get

$$
\left\|\delta \lambda_{y}(t)\right\| \leq C_{\kappa}\left(e^{-\kappa t}+e^{-\kappa\left(T_{f}-t\right)}\right), \quad \forall t \in\left[0, T_{f}\right]
$$

Note that there exists $R=R(\mathfrak{r})$ s.t. $\|\delta y\|+\left\|\delta \lambda_{y}\right\| \leq R$. Then, using additional calculations for $\delta w(t)$, we get,

$$
\begin{aligned}
& \|\delta w(t)\|=\left\|w\left(y, \lambda_{y}\right)-\bar{w}\right\| \leq \\
& \sup _{B\left(\left(\bar{y}, \bar{\lambda}_{y}\right), R\right)}\left\|\nabla_{y} w\right\|\|\delta y\|+\sup _{B((\bar{y}, \bar{\lambda}}\left\|\nabla_{\lambda_{y}} w\right\|\left\|\delta \lambda_{y}\right\|,
\end{aligned}
$$

for all $t \in\left[0, T_{f}\right]$. Combining (28), (29) and (30) leads us to the exponential turnpike estimate given in (19) and thus concludes the proof of Theorem 1 .

\section{Application to a higher dimensional system}

\subsection{Algal competition using Droop's model}

In this section, we focus on a model of competition between two microalgae populations in dimension 5 , with the aim of achieving species separation over a fixed time window. We tweak this issue using a system reduction in order to rewrite the optimization objective in term of the generic OCP (2). Let $x_{1}$ and $x_{2}$ be the biomass concentrations of two different strains of microalgae, initially coexisting in a bioreactor with one limiting substrate $(s)$, s.t., $s(t) \in\left[0, s_{i n}\right]$, for all $t \geq 0$, where $s_{i n}$ is the constant substrate influent concentration. Each microalgae species uptakes the free nutrient $s$. The intracellular amount of this limiting element is the cell quota $q_{i}$, with $i=1,2$.

A general class of quota models, including the classical Droop's model (Droop (1968)), writes,

$$
\left\{\begin{array}{l}
\dot{s}=\left(s_{i n}-s\right) D-\sum_{i=1}^{2} \rho_{i}(s) x_{i} \\
\dot{q}_{i}=\rho_{i}(s)-\mu_{i}\left(q_{i}\right) q_{i} \\
\dot{x}_{i}=\left[\mu_{i}\left(q_{i}\right)-D\right] x_{i}
\end{array}\right.
$$


The function $\mu_{i}$ in $(31)$ stands for the growth rate of the $i$-th species, while $\rho_{i}$ is its nutrient uptake rate. The dilution rate $D$ and the substrate input $s_{i n}$ can generally be manipulated. The functions $\rho_{i}$ and $\mu_{i}$ are nonnegative and increasing bounded functions, s.t., $0 \leq \rho_{i}(s) \leq \rho_{m i}$, and, $0 \leq \mu_{i}\left(q_{i}\right) \leq \mu_{m i}$, where $\rho_{m i}$ and $\mu_{m i}$ are positive constants. Typically in Droop's model, the uptake rate $\rho_{i}(s)$ is expressed in terms of Michaelis-Menten kinetics,

$$
\rho_{i}(s)=\frac{\rho_{m i} s}{K_{s i}+s},
$$

where $K_{s i}$ is a positive constant. The most classical form -known as Droop's growth- for $\mu_{i}$ is given for $i=1,2$ by,

$$
\mu_{i}\left(q_{i}\right)=\mu_{i \infty}\left(1-\frac{Q_{i \min }}{q_{i}}\right), \quad q_{i} \geq Q_{i \min }
$$

The minimum intracellular quota $Q_{i \text { min }}>0$ defines the quota under which cell division cannot occur. Other forms have been used to describe the growth rate such as the Caperon-Meyer equation (Caperon \& Meyer (1972)),

$$
\mu_{i}\left(q_{i}\right)=\frac{\mu_{i \infty}\left(q_{i}-Q_{i \min }\right)}{q_{i}-Q_{i \min }+k_{q_{i}}}, q_{i} \geq Q_{i \min }, k_{i q}>0
$$

another possible form that we also use in this work is,

$$
\mu_{i}\left(q_{i}\right)=\frac{\mu_{i \infty} q_{i}^{n_{i}}}{q_{i}^{n_{i}}+k_{q_{i}}^{n_{i}}}, \quad n_{i}>1
$$

Notice that the choices (34)-(35) lead to nonlinear terms $\mu_{i}\left(q_{i}\right) q_{i}$ in (31). In all these cases, the resulting quota is bounded for all $t \geq 0$, i.e., $Q_{i \min } \leq q_{i}(t) \leq q_{m i}$, where $q_{m i}$ is the maximum internal storage rate (Bernard (2011)). In the sequel, the quota variables $q_{i}$ will stand for $y_{i}$ in model (1) and we will prove that: $(\boldsymbol{i})$ OCP (2) achieves microalgae separation, through maximization of relative concentration $x_{1}\left(T_{f}\right) / x_{2}\left(T_{f}\right)$, (ii) Theorem 1 applies for different functions $\mu_{i}$ (given by (33)-(35)), i.e., turnpike-type behaviors characterize microalgae selection under various growth rates.

\subsection{Controlling the substrate concentration}

Now, let us assume that the free substrate concentration $s$ is controlled by an ad'hoc controller.For instance, by playing with the concentration of the influent substrate $s_{i n}$ and with the dilution rate $D$, the concentration $s$ can follow a desired (smooth enough) reference trajectory $s(t)=s^{*}(t)$. Different control designs can be used such as linearizing control (Bastin (1990)), asymptotic control (Mailleret et al. (2004)) or other approaches that demonstrated their efficiency on microalgae (see, e.g., Bernard et al. (2015); Tebbani et al. (2014)). By assuming a perfect tracking of the substrate towards $w(t)$, the system (31) then reduces to,

$$
\left\{\begin{array}{l}
\dot{q}_{i}=\rho_{i}(w)-\mu_{i}\left(q_{i}\right) q_{i} \\
\dot{x}_{i}=\left[\mu_{i}\left(q_{i}\right)-D\right] x_{i}
\end{array}\right.
$$

This system is triangular, and the dynamics of $x_{i}$ results from the dynamics of $q_{i}$. Consequently, we can focus exclusively on the quota-dynamics $q_{i}$, for $i=1,2$.

\subsection{Maximizing the relative biomass density}

Species separation is achieved when maximizing the relative density of the species of interest $x_{1}$ at $T_{f}$, i.e., finding the optimal control maximizing the quantity,

$$
\tilde{\mathcal{C}}\left(T_{f}\right)=\frac{x_{1}\left(T_{f}\right)}{x_{2}\left(T_{f}\right)} .
$$

Let us state the following key result to end up with a functional that does not depend on the states $x_{i}$, but still maximizes the relative concentration at the final-time.

Proposition 1 Any solution of the class of systems (36) maximising $\mathcal{C}_{\left[0, T_{f}\right]}$ defined in OCP $(2)$ is equivalently maximizing $\tilde{\mathcal{C}}_{T_{f}}$ in (37), where the functions $f_{i}$ in $O C P$ (2) stand for the growth rates $\mu_{i}$ in system (36).

Proof: Indeed, the cost function in OCP (2) writes, $\mathcal{C}_{\left[0, T_{f}\right]}=\int_{0}^{T_{f}}\left[\mu_{1}\left(q_{1}(t)\right)-D\right]-\left[\mu_{2}\left(q_{2}(t)\right)-D\right] d t$. Using the state dynamics, $\mu_{i}\left(q_{i}\right)-D=\frac{\dot{x}_{i}}{x_{i}}$, we get, $\mathcal{C}_{\left[0, T_{f}\right]}=\left[\ln \left(x_{1}(t)\right)-\ln \left(x_{2}(t)\right)\right]_{0}^{T_{f}}=\ln \left(\frac{x_{1}\left(T_{f}\right) x_{2}(0)}{x_{1}(0) x_{2}\left(T_{f}\right)}\right)$. Thus, maximizing $\mathcal{C}_{\left[0, T_{f}\right]}$ indeed consists in maximizing $\tilde{C}_{T_{f}}$. The advantage of considering the criterion $\mathcal{C}_{\left[0, T_{f}\right]}$ instead of the natural criterion $\tilde{\mathcal{C}}_{T_{f}}$ is that it is independent of the dynamics of the states $x_{i}$, for $i=1,2$.

Thus, the optimization problem can now be reduced to an OCP as in (2), involving the $\operatorname{cost} \mathcal{C}_{\left[0, T_{f}\right]}$ and associated with the reduced-system (in the form (1)),

$$
\left\{\begin{array}{l}
\dot{q}_{1}=-\mu_{1}\left(q_{1}\right) q_{1}+\rho_{1}(w) \\
\dot{q}_{2}=-\mu_{2}\left(q_{2}\right) q_{2}+\rho_{2}(w)
\end{array}\right.
$$

where the growth rate $\mu_{i}$ is given by one of the functions (33)-(35), the uptake rate $\rho_{i}$ is given by (32), and the control $w$ is equivalent to the substrate $s$ in (31) once regulated by the ad'hoc controller. This is especially true since (1) in Theorem 1 states that the solution of the OCP (2) corresponds to the singular control function with no bangs over the time-window $\left[0, T_{f}\right]$, and consequently the optimal control $w^{\star}(t)$ has thus the required smoothness to be tracked in the original system (31) through the ad'hoc controller. To sum up, we can see that finding a control for system (31) approaching as close as possible the solution $w^{\star}(t)$ of the OCP studied in Sections 2-3 will provide an excellent suboptimal control for the OCP of the ful-system (31) associated with the criterion (37). 


\subsection{Optimal synthesis of species separation}

Now, we are ready to apply the results obtained in Sect. 2-3 to the problem of species selection. Firstly, the staticOCP associated with (38) and $\mathcal{C}_{\left[0, T_{f}\right]}$ states,

$\left\{\begin{array}{l}\underset{w \in\left[0, w_{m}\right]}{\operatorname{Maximize}} \Delta(w)=\mu_{1}\left(q_{1}(w)\right)-\mu_{2}\left(q_{2}(w)\right), \\ \text { where }-\mu_{i}\left(q_{i}\right) q_{i}+\rho_{i}(w)=0 \text { for } i=1,2,\end{array}\right.$

under the steady-state constraints $-\mu_{i}\left(q_{i}\right) q_{i}+\rho_{i}(w)=0$ for $i=1,2$. Similarly to Section 2, the solution of (39) -including the co-states- is denoted by, $\mathcal{E}^{\text {static }}=\left(\bar{q}_{1}, \bar{q}_{2}, \bar{\lambda}_{q_{1}}, \bar{\lambda}_{q_{2}}, \bar{w}\right)$. This optimal steady-state satisfies (9)-(10), using this time the quota-coordinates in (38), under the standard assumption of Section 2, i.e., $\bar{w} \in\left(0, w_{m}\right)$ as in Fig. 1 , which is generally satisfied for $\rho_{i}$ and $\mu_{i}$ in (32)-(35), as illustrated later in numerical simulations. A case in point is the classical Droop's model associated with (32)-(33), in which case the function $\Delta$ rewrites,

$$
\Delta(w)=\mu_{1}\left(\delta_{1}^{-1}(w)\right)-\mu_{2}\left(\delta_{2}^{-1}(w)\right)
$$

where, $\delta_{i}(\ell)=\rho_{i}^{-1}\left(\mu_{i}(\ell) \ell\right)$, and since, $\rho_{i}^{-1}(\ell)=\frac{K_{i} \ell}{\rho_{i m}-\ell}$, for all $\ell \in\left[0, \rho_{i m}\right)$, we obtain, $\delta_{i}(\ell)=K_{i} \frac{\ell-Q_{i \min }}{\sigma_{i}-\ell}$, where, $\sigma_{i}=\frac{\rho_{i m}}{\mu_{i \infty}}+Q_{i \min }$. This leads to, $\delta_{i}^{-1}(\ell)=\frac{\sigma_{i} \ell+K_{i} Q_{i \min }}{\ell+K_{i}}$, and consequently we end up in the Droop's case with,

$$
\mu_{i}\left(\delta_{i}^{-1}(\ell)\right)=\frac{\rho_{i m} \ell}{\sigma_{i} \ell+K_{i} Q_{i \min }}, \quad i=1,2 .
$$

Using (41) we can compute successive derivatives of $\Delta(w)$ in the Droop's case, i.e., $\Delta^{\prime}(w)=\frac{\rho_{1 m} K_{1} Q_{1 \min }}{\left(K_{1} Q_{1 \min }+\sigma_{1} w\right)^{2}}-$ $\frac{\rho_{2 m} K_{2} Q_{2 \min }}{\left(K_{2} Q_{2} \min +\sigma_{2} w\right)^{2}}$, and, $\Delta^{\prime \prime}(w)=\varkappa_{1}(w)-\varkappa_{2}(w)$, where, $\varkappa_{i}(w)=-\frac{2 K_{i} \mu_{i \infty}^{2} Q_{i \min } \rho_{i m}\left(\mu_{i \infty} Q_{i \min }+\rho_{i m}\right)}{\left(K_{i} \mu_{i \infty} Q_{i \min }+\left(\mu_{i \infty} Q_{i \min }+\rho_{i m}\right) w\right)^{3}}$. Thus, by looking for the points that satisfy $\Delta^{\prime}(\bar{w})=0$ and $\Delta^{\prime \prime}(\bar{w})<0$ in the case where $\mu_{i}$ is given by $(33)$, we deduce that the steady-state $\mathcal{E}^{\text {static }}=\left(\bar{w}, \bar{q}_{1}, \bar{q}_{2}, \bar{\lambda}_{q_{1}}, \bar{\lambda}_{q_{2}}\right)$, satisfies, $\bar{q}_{i}=\frac{1}{\mu_{i \infty}}\left(\rho_{i}(\bar{w})+\mu_{i \infty} Q_{i \text { min }}\right)$, for $i=1,2$, $\bar{\lambda}_{q_{1}}=-\frac{Q_{1 \min }}{\bar{q}_{1}^{2}}$, and, $\bar{\lambda}_{q_{2}}=\frac{Q_{2 \min }}{\bar{q}_{2}^{2}}$. Numerically, we check that only one numerical value $\bar{w}$ belongs to $\left(0, w_{m}\right)$ for a sufficiently large $w_{m}$, leading then to the configuration in Fig. 1 (Fig. 4 in Droop's case). We refer to Appendices A-B in Djema et al. (2021) for more details on the practical determination of $\mathcal{E}^{\text {static }}$ in the Droop's case. In a similar way, we can determine the analytic expression of $\mathcal{E}^{\text {static }}$ for different growth-rates $((34)-(35))$. Therefore, under suitable conditions a unique $\mathcal{E}^{\text {static }}$ exists and satisfies $\bar{w} \in\left(0, w_{m}\right)$, and thus in light of Theorem 1 we derive the following statement as a direct consequence.

Claim 1 (Application of Theorem 1) Let fix any biological parameters for the functions $\rho_{i}$ in (32) and $\mu_{i}$ in (33)-(35), $i=1,2$, in system (31), s.t., there exists a unique $\mathcal{E}^{\text {static }}$ with $\bar{w} \in\left(0, w_{m}\right)$, solution of the staticOCP (39) then,

(1) the optimal control $w^{\star}(t)$ for all $t \in\left[0, T_{f}\right]$ is singular, i.e., it does not involve bang arcs 0 and $w_{m}$ over $\left[0, T_{f}\right]$. (2) Moreover, if the corresponding conditions (17) and (18) are fulfilled, the singular optimal control (substrate concentration) $w^{\star}(t)$ and its related quota $q_{i}(t)$, as well as their respective co-states $\lambda_{q_{i}}(t), i=1,2$, exhibit over the fixed time-horizon $\left[0, T_{f}\right]$ an exponential turnpike behavior characterized in (19).

Note that the overlapping of favorable features (namely the facts that $\bar{w}$ solution of the static-OCP is remote from the bounds 0 and $w_{m}$, the optimal control is inherently singular, i.e., $w_{s}(t) \in\left(0, w_{m}\right)$ for all $t \in\left[0, T_{f}\right]$, and also the exponential turnpike keeping $w_{s}(t)$ almost constant most of the time) results in strong smoothness of the optimal control and the optimal trajectories, as illustrated in the next section. Nonetheless, this smoothness does not imply that the resulting ad'hoc controller of system (31) should be smooth as well, as shown in Djema et al. (2020b) where the obtained dilution-based control is of type bang-singular. To sum up the methodology provided in this paper: the first step is to determine the optimal point $\mathcal{E}^{\text {static }}$ s.t. $\bar{w} \in\left(0, w_{m}\right)$, solution of the static-OCP corresponding to a given dataset of biological parameters and functions in the system (31). Next, we compute the matrix $\Psi$ defined around $\mathcal{E}^{\text {static }}$ as in (14). Then, the conditions stated in (2)-Theorem 1 ensure the existence of a turnpike-type behavior of the optimal solution of the reduced system (1). The numerical computation of the eigenvalues $\kappa_{j}, j=1, \ldots, 4$, which are defined in (15), is illustrated in different cases of the functions $\mu_{i}$ in Section 5.5. Finally, it is worth noting that the conditions (17) in Theorem 1 appear to be systematically satisfied in numerical simulations, leading accordingly to a turnpike behavior in all the evaluated cases.

\subsection{Numerical synthesis through direct methods}

The direct-optimization approach performed in this section illustrates the results of Theorem 1 applied to the quota-model in Sect. 5 (System (38)) for the different growth functions ((33)-(35)) illustrated in Fig. 2.

In this perspective, the state variables and the control function of system (38) are discretized, by setting a subdivision of the time-horizon $\left[0, T_{f}\right]$ with fixed $T_{f}$, and the substrate-control $w$ is discretized over $\mathcal{W}$. The state dynamics $q_{1}$ and $q_{2}(38)$ is discretized with Lobatto scheme (Betts (2010)), i.e. RK method of type Lobatto-IIIC, based on an implicit trapezoidal rule, of order 6 . suitable for stiff-nonlinear problems. Thus, numerical direct methods transform OCP (2) (stated in Sect. 2, associated with System (38)) through the discretization step, into a nonlinear programming problem in finitedimension (see Djema et al. (2021), Sect. 5.5). Using the numerical values of the biological parameters in Fig. 2, 

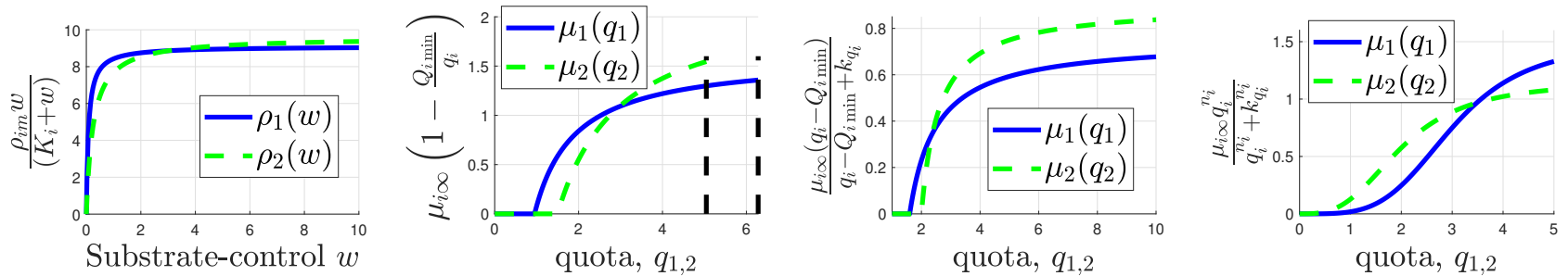

Fig. 2. Various functions used in Droop and Caperon-Meyer models (Caperon \& Meyer (1972); Droop (1968)). From left to right: absorption functions $\rho_{i}$ in $(32)$ with, $\rho_{1 m}=9.1\left(\mu m o l . L^{-1} . d^{-1}\right), K_{1}=0.08\left(\mu m o l . L^{-1}\right) \rho_{2 m}=9.6\left(\mu m o l . L^{-1} . d^{-1}\right)$, $K_{2}=0.25\left(\mu \mathrm{mol} . \mathrm{L}^{-1}\right)$. Growth rate $\mu_{i}$ in (33) with, $\mu_{1 \infty}=1.6 \mathrm{day}{ }^{-1}, Q_{1 \min }=0.95 \mu \mathrm{mol} / \mathrm{L}, \mu_{2 \infty}=2.2 \mathrm{day}{ }^{-1}$, $Q_{2 \min }=1.5 \mu \mathrm{mol} / \mathrm{L}$. Growth rate $\mu_{i}$ in $(34)$ with, $\mu_{1 \infty}=0.75 \mathrm{day}{ }^{-1}, Q_{1 \min }=1.6 \mu \mathrm{mol} / \mathrm{L}, k_{q 1}=0.9 \mu \mathrm{mol} / \mathrm{L}, \mu_{2 \infty}=0.9 \mathrm{day}-1$, $Q_{2 \mathrm{~min}}=2 \mu \mathrm{mol} / L, k_{q 2}=0.6 \mu \mathrm{mol} / \mathrm{L}$. Growth rate $\mu_{i}$ in $(35)$ with, $\mu_{1 \infty}=1.5 \mathrm{day}^{-1}, n_{1}=4, k_{q 1}=3 \mu \mathrm{mol} / \mathrm{L}, \mu_{2 \infty}=1.15 \mathrm{day}-1$, $n_{2}=3, k_{q 2}=2 \mu \mathrm{mol} / L$.

\begin{tabular}{|c|c|c|c|}
\hline & Example 1 & Example 2 & Example 3 \\
\hline $\bar{w}$ & 0.021 & 0.015 & 0.074 \\
$\bar{q}_{1}$ & 2.164 & 0.015 & 3.91 \\
$\bar{q}_{2}$ & 1.84 & 0.015 & 2.69 \\
$\bar{\lambda}_{q_{1}}$ & 0.44 & 0.322 & 0.173 \\
$\bar{\lambda}_{q_{2}}$ & -0.20 & -0.1377 & 2.690 \\
$\kappa_{1,2}$ & $2.332 \pm 0.81 i$ & $1.74 \pm 1.09 i$ & \pm 3.02 \\
$\kappa_{3,4}$ & $-2.332 \pm 0.81 i$ & $-1.74 \pm 1.09 i$ & \pm 0.82 \\
\hline Table 1
\end{tabular}

Values of the static steady-state $\mathcal{E}^{\text {static }}$ and eigenvalues of the matrix $\Psi$ defined at $\mathcal{E}^{\text {static }}$ for the 3 examples.

we performed several direct optimizations in Bocop with appropriate settings (tolerance for NLP solver: $10^{-34}$, 2000 time-steps). No issue is encountered regarding the initialization and starting points for solving the studied problem. In all the following examples, the conditions of Theorem 1 are satisfied, and the optimal solutions satisfy the turnpike property (19) as stated in Claim 1.

Example 1 We set $T_{f}=15$ days and we consider that the growth functions $\mu_{i}$ are in the Droop's form (33). In this case, the sufficient conditions given in Trélat \& Zuazua (2015) are not satisfied (see Remark 3). The function $\Delta(w)=\mu_{1}\left(\delta_{1}^{-1}(w)\right)-\mu_{2}\left(\delta_{2}^{-1}(w)\right)$ is given in Fig. (3). In addition, the static steady-state $\mathcal{E}^{\text {static }}$ and the eigenvalues of the matrix $\Psi$ are given in Tab. . Notice also that in this case $H_{y y}=\operatorname{diag}\{0.2997,-1.0443\}$. According to Theorem 1 (see Claim 1), the turnpike property (19) holds as shown in Fig. 4.

Example 2 We set $T_{f}=30$ days and we consider that the growth functions $\mu_{i}$ are in the form (34), with the biological parameters given in Fig. 2. Results are in Tab. 1. The turnpike holds according to Theorem 1, as described in Claim 1 and illustrated in Fig. 5. Notice that the sufficient conditions for the turnpike existence given in Trélat $\&$ Zuazua (2015) are not satisfied, since $H_{y y}=$ $\operatorname{diag}\{0.116993,-0.939631\}$.

Example 3 We set $T_{f}=50$ days. The growth functions $\mu_{i}$ are in the form (35), with the biologi-

cal parameters given in Fig. 2. Results are in Tab. 1. Similarly to the previous examples, here we get, $H_{y y}=\operatorname{diag}\{0.183898,-0.210028\}$. However, the turnpike characteristics hold in the optimal solution according to Theorem 1 and Claim 1, as shown in Fig. 6.

In all these examples (Fig. 4-6), the optimal solutions of the OCP $(2)$, settled in relatively large time $\left[0, T_{f}\right]$, consist of three pieces: the first and the last ones are relatively short arcs, and the middle piece being a relatively long-time arc staying close to the optimal steady-state solution of the associated static-OCP (8).

\section{Conclusion}

The optimal control has been fully-characterized through a complete study combining Pontryagin's principle and turnpike frameworks relying upon classical hyperbolic system properties. The turnpike theory (Trélat \& Zuazua (2015); Pighin \& Porretta (2016)) considerably simplifies the understanding of the control strategy and provides a clear insight into the optimal control structure. By investigating the OCP and its associated static version, the main result in this paper proves that the optimal control is always singular and provides sufficient conditions ensuring the existence of turnpike behaviors characterizing the optimal trajectories. We had to upgrade the existing turnpike results so that they can apply to the specificity of our models. The considered class of systems naturally appears in the framework of competition between two microbial or algal species, but a similar structure is likely to play a role for other classes of problems. In general, solving an OCP in dimension 5 is a challenging issue (see Djema et al. $(2020 \mathrm{a}, \mathrm{b}))$. Thus, the microalgae optimal selection problem was reduced in this paper considering the core dynamics in dimension 2 . This reduction approach using a back-stepping philosophy might also prove to be efficient in other contexts (see, e.g., Yegorov et al. (2019); Caillau et al. (2020)), and this will be the main focus of our future work.

Acknowledgements: This work was supported by the ADEME BioMSA and Maximic ANR projects in France. The authors 

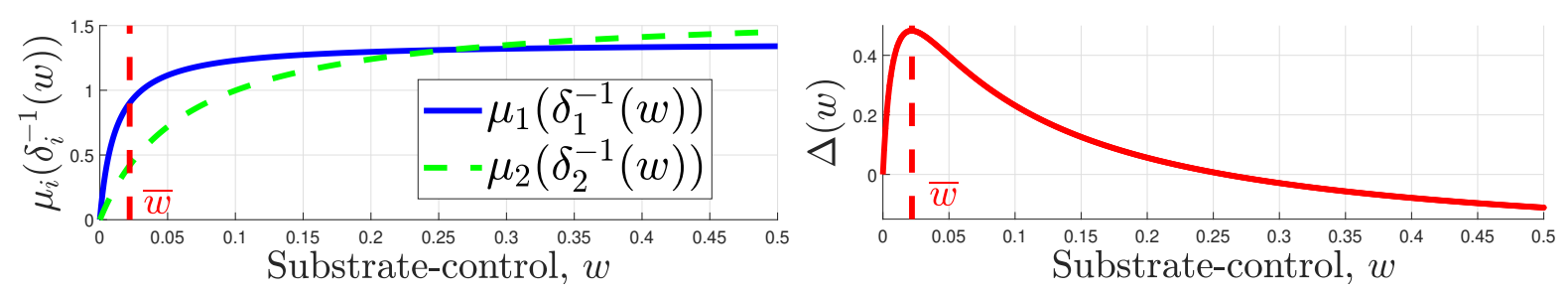

Fig. 3. The functions $\mu_{i}\left(\delta_{i}^{-1}(w)\right)$ and $\Delta(w)$ using the data given in Fig. 2 with $\mu_{i}$ as in (33).
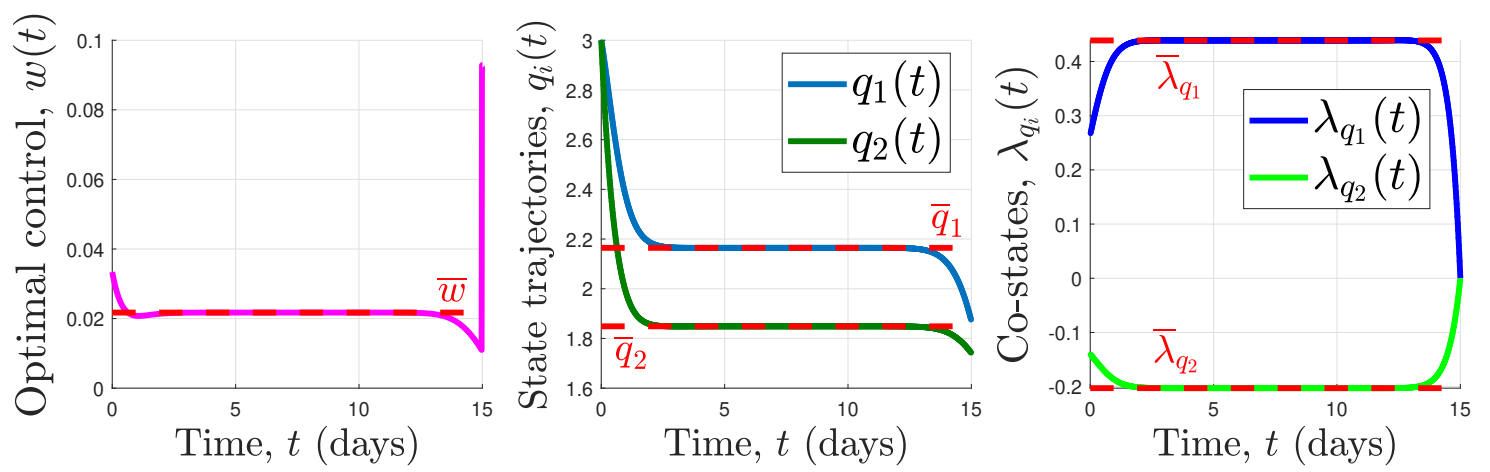

Fig. 4. The optimal control and optimal trajectories given by Bocop in Example 1.
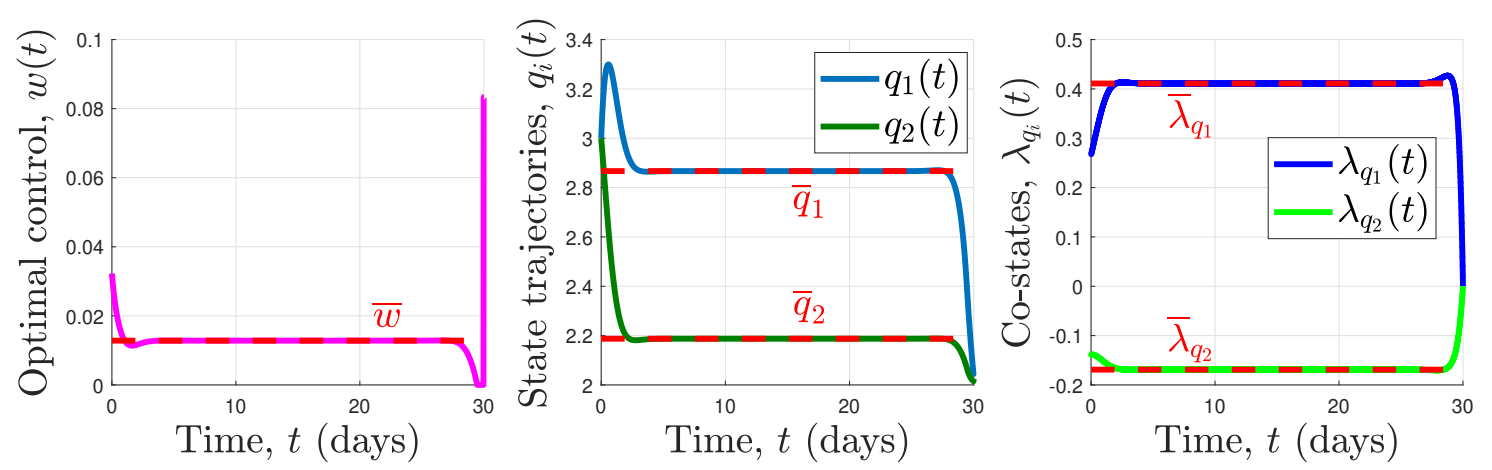

Fig. 5. The optimal control and optimal trajectories given by Bocop in Example 2.
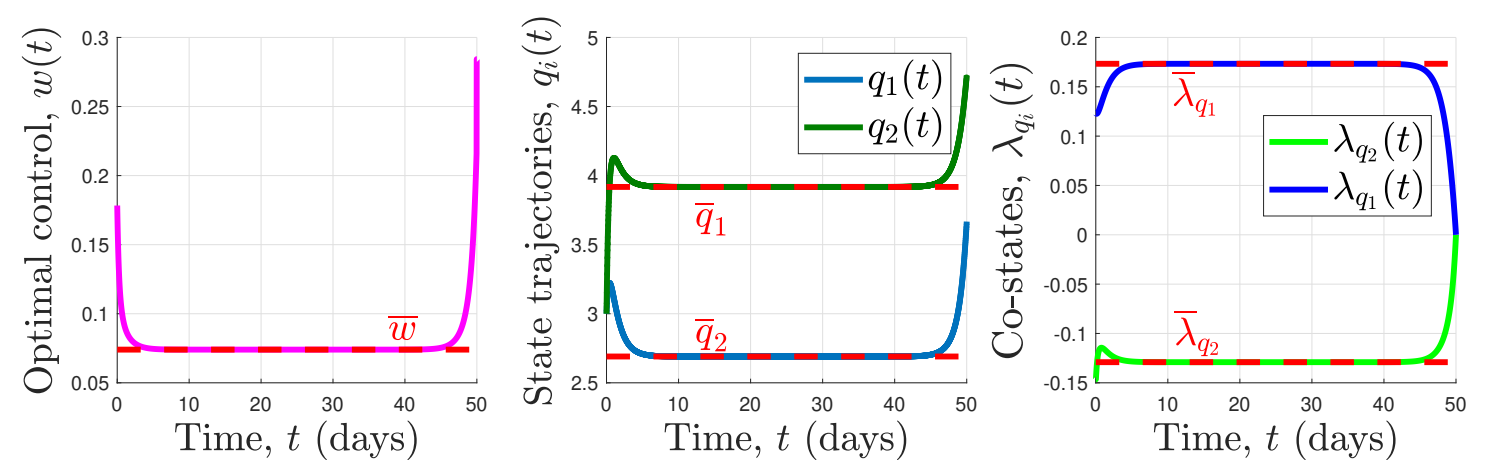

Fig. 6. The optimal control and optimal trajectories given by Bocop in Example 3 .

acknowledge J.-B. Caillau, J.-B. Pomet and J.-L. Gouzé for the stimulating discussions on this topic.

\section{References}

P.F.D Almeida, R.S. Moreira, R.C.D.C. Almeida, A.K. Guimaraes, A.S. Carvalho, C. Quintella, M.C.A. Esperidia, 
C.A. Taft, Selection and application of microorganisms to improve oil recovery., Eng. in life Sci., 4(4), pp.319-325., (2004).

A.F. Aravantinou, M.A. Theodorakopoulos, I.D. Manariotis, Selection of microalgae for wastewater treatment and potential lipids production., Bioresource tech., 147, pp.130-134., (2013).

C. Baroukh, R. Muñoz-Tamayo, J.P. Steyer, O. Bernard, A state of the art of metabolic networks of unicellular microalgae and cyanobacteria for biofuel production., Metabolic engineering, 30, pp.49-60., (2015).

T. Bayen, \& F. Mairet, Optimization of the separation of two species in a chemostat. Automatica, 50(4), p.6, (2014).

T. Bayen, \& F. Mairet, Optimization of strain selection in evolutionary continuous culture. International Journal of Control, 90(12), pp. 2748-2759, (2017).

O. Bernard, F. Mairet, B. Chachuat, Modelling of microalgae culture systems with applications to control and optimization. Microalgae Biotechnology. Springer, Cham, pp.59-87, (2015).

O. Bernard, Hurdles and challenges for modelling and control of microalgae for $\mathrm{CO}_{2}$ mitigation and biofuel production. Journal of Process Control 21, no. 10, pp. 1378-1389, (2011).

J.T. Betts, Practical methods for optimal control and estimation using nonlinear programming. Siam, Advances in Design \& Control, 2nd Edition, Vol. 19, p. 427, (2010).

L.T. Biegler, Nonlinear Programming: Concepts, Algorithms, and Applications to Chemical Processes. MPS-SIAM Series on Optimization (Book 10), SIAM-Society for Industrial and Applied Mathematics, p. 415, (2010).

H. Bonnefond, G. Grimaud, J. Rumin, G. Bougaran, A. Talec, M. Gachelin, M. Boutoute, E. Pruvost, O. Bernard, A. Sciandra, Continuous selection pressure to improve temperature acclimation of Tisochrysis lutea., PloS one, 12(9), (2017).

F.J. Bonnans, D. Giorgi, V. Grelard, B. Heymann, S. Maindrault, P. Martinon, O. Tissot, J. Liu, BOCOP: an open source toolbox for optimal control - A collection of examples. Team Commands, Inria Technical Reports, http://bocop.org, (2017).

J.-B. Caillau, W. Djema, L. Giraldi, J.-L. Gouzé, S. Maslovskaya, J.-B. Pomet. The turnpike property in maximization of microbial metabolite production., Abstract IFAC WC, (2020).

J. Caperon, \& J. Meyer, Nitrogen-limited growth of marine phytoplankton - II. Uptake kinetics and their role in nutrient limited growth of phytoplankton. In Deep Sea Research and Oceanographic Abstracts, Vol. 19, No. 9, pp. 619-632, (1972).

L. Cesari, Optimization - Theory and applications, Problems with ordinary differential equations., Vol. 17 of Applications of Mathematics (New York), Springer-Verlag, New York, (1983).

Y. Chisti, Biodiesel from microalgae., Biotechnology Advances, Vol.25 (3), pp.294-306., (2007).

W. Djema, L. Giraldi, O. Bernard, An Optimal Control Strategy Separating Two Species of Microalgae in Photobioreactors. IFAC, Conference on Dynamics and Control of Process Systems, including Biosystems-12th DYCOPS., (2019).

W. Djema, L. Giraldi, S. Maslovskaya, O. Bernard, Turnpike features in optimal selection of species represented by quota models: extended proofs., [Research Report] RR-9399, Inria Sophia Antipolis. 2021, pp.29. 〈hal-03253160〉, (2021).

W. Djema, O. Bernard, T. Bayen, Optimal control separating two microalgae species competing in a chemostat. Proceed. IEEE Conference on Decision \& Control CDC, (2020).

W. Djema, O. Bernard, L. Giraldi, Separating Two Species of Microalgae in Photobioreactors in Minimal Time., Journal of Process Control (JPC) Vol. 87, pp. 120-129, https ://doi.org/10.1016/j.jprocont.2020.01.003, (2020).

D. Dochain, \& G. Bastin, On-line estimation and adaptive control of bioreactors., Vol. 1. Elsevier, (1990).

M.R. Droop, Vitamin $B_{12}$ and marine ecology. IV. The kinetics of uptake growth and inhibition in Monochrysis lutheri. J. Mar. Biol. Assoc. 48 (3), pp. 689-733, (1968).

M.R. Droop, 25 years of algal growth kinetics, a personal view. Bot. Mar. 16, pp. 99-112, (1983).

M. Gachelin, M. Boutoute, G. Carrier, A. Talec, E. Pruvost, F. Guihéneuf, O Bernard, A. Sciandra, Enhancing PUFA-rich polar lipids in Tisochrysis lutea using adaptive laboratory evolution (ALE) with oscillating thermal stress., Applied Microbiology and Biotechnology, pp.1-12., Springer, (2020).

M. Gugat, \& F.M. Hante, On the turnpike phenomenon for optimal boundary control problems with hyperbolic systems., SIAM J. on Cont. and Opt., 57(1), pp.264-289., (2019).

I.E. Huertas, M. Rouco, V. Lòpez-Rodas, E. Costas, Estimat- ing the capability of different phytoplankton groups to adapt to contamination: herbicides will affect phytoplankton species differently., New Phytologist, 188(2), pp.478-487., (2010).

J. Lohéac, E. Trélat, E. Zuazua, Minimal controllability time for finite-dimensional control systems under state constraints., Automatica, 96, pp.380-392., (2018).

L. Mailleret, O. Bernard, J-P Steyer, Nonlinear adaptive control for bioreactors with unknown kinetics., Automatica 40.8, pp.1379-1385, (2004).

B.P. Molinari, The time-invariant linear-quadratic optimal control problem. Automatica, 13(4), pp.347-357., (1977).

L. Rodolfi, G. Chini Zittelli, N. Bassi, G. Padovani, N. Biondi, G. Bonini, M.R. Tredici, Microalgae for oil: Strain selection, induction of lipid synthesis and outdoor mass cultivation in a low-cost photobioreactor., Biotechnology and bioengineering, 102(1), pp.100-112., (2009).

E.C. Odjadjare, T. Mutanda, A.O. Olaniran, Potential biotechnological application of microalgae: a critical review., Critical Reviews in Biotechnology, 37(1), pp.37-52., (2017).

D. Pighin, \& A. Porretta, Long time behaviour of Optimal Control problems and the Turnpike Property, Thesis Università degli Studi di Roma Tor Vergata, cmc.deusto.eus, pp.205, (2016).

A. Porretta, \& E. Zuazua, Long time versus steady state optimal control., SIAM J. Cont. \& Opti., 51(6), pp.4242-4273., (2013).

L.S. Pontryagin, V.G. Boltyanskiy, R.V. Gamkrelidze, E.F. Mishchenko, Mathematical theory of optimal processes. New York, NY Springer, (1964)

H.L. Smith, \& P. Waltman, The theory of the chemostat: $d y$ namics of microbial competition. Cambridge Studies in Mathematical Biology, Cambridge Univ. Press, (1995).

W. Swenson, D.S. Wilson, R. Elias, Artificial ecosystem selection., Proceedings of the National Academy of Sciences, 97(16), pp.9110-9114., (2000).

S. Tebbani, R. Filali, F. Lopes, D. Dumur, D. Pareau, CO2 Biofixation by Microalgae: Modeling, estimation and control., John Wiley \& Sons., (2014).

E. Trélat, C. Zhang, Integral and measure-turnpike properties for infinite-dimensional optimal control systems., Mathematics of Control, Signals \& Systems, 30(1), p.3., (2018).

E. Trélat, \& E. Zuazua, The turnpike property in finitedimensional nonlinear optimal control., Journal of Differential Equations, 258(1), pp.81-114., (2015).

M.J. Walsh, L.G. Van Doren, D.L. Sills, I. Archibald, C.M. Beal, X.G. Lei, M.E. Huntley, Z. Johnson, C.H. Greene, Algal food and fuel coproduction can mitigate greenhouse gas emissions while improving land and water-use efficiency., Environmental Research Letters, 11(11), p.114006., (2016).

W. Wiechert, Modeling and simulation: tools for metabolic engineering., Journal of biotechnology, 94(1), pp.37-63., (2002).

R.H. Wijffels, \& M.J. Barbosa An outlook on microalgal biofuels., Science 329, (5993), pp.796-799., (2010).

I. Yegorov, F. Mairet, H. de Jong, J.L. Gouzé, Optimal control of bacterial growth for the maximization of metabolite production., J. of Math. Bio, 78(4), pp.985-1032., (2019).

C. Yoo, S.Y. Jun, J.Y. Lee, C.Y. Ahn, H.M. Oh, Selection of microalgae for lipid production under high levels carbon dioxide., Bioresource technology, 101(1), pp.S71-S74., (2010).

A.J. Zaslavski, Turnpike theory of continuous-time linear optimal control problems., Vol. 104. New York: Springer., (2015). 